\title{
THE NON-STEROIDAL ANTI-INFLAMMATORY DRUG CARPROFEN NEGATIVELY IMPACTS NEW BONE FORMATION AND ANTIBIOTIC EFFICACY IN A RAT MODEL OF ORTHOPAEDIC-DEVICE-RELATED INFECTION
}

\author{
M-A. Burch ${ }^{1}$, A. Keshishian ${ }^{1}$, C. Wittmann ${ }^{1}$, D. Nehrbass ${ }^{1}$, U. Styger ${ }^{1}$, G. Muthukrishnan ${ }^{2}$, D. Arens ${ }^{1}$, \\ V.A. Stadelmann ${ }^{1,3}$, R.G. Richards ${ }^{1}$, T.F. Moriarty ${ }^{1}$ and K. Thompson ${ }^{1, *}$ \\ ${ }^{1}$ AO Research Institute Davos, Davos, Switzerland \\ ${ }^{2}$ Centre for Musculoskeletal Research, Department of Orthopaedics, University of Rochester School of \\ Medicine, 601 Elmwood Avenue, Box 665, Rochester, NY, 14642, USA \\ ${ }^{3}$ Department of Teaching, Research and Development, Schulthess Clinic, Zürich, Switzerland
}

\begin{abstract}
Non-steroidal anti-inflammatory drugs (NSAIDs) are widely used for pain management during recovery from orthopaedic surgery. NSAID use is associated with increased risk of bone healing complications but it is currently unknown whether NSAIDs increase the risk of developing an orthopaedic-device-related infection (ODRI) and/or affect its response to antibiotic therapy. The present study aimed to determine if administration of the NSAID carprofen [a preferential cyclooxygenase-2 (COX-2) inhibitor] negatively affected Staphylococcus epidermidis (S. epidermidis) bone infection, or its subsequent treatment with antibiotics, in a rodent ODRI model.

Sterile or S. epidermidis-contaminated screws $\left(\sim 1.5 \times 10^{6} \mathrm{CFU}\right)$ were implanted into the proximal tibia of skeletally mature female Wistar rats, in the absence or presence of daily carprofen administration. A subset of infected animals received antibiotics (rifampicin plus cefazolin) from day 7 to 21, to determine if carprofen affected antibiotic efficacy. Bone changes were monitored using in vivo $\mu$ CT scanning and histological analysis. The risk of developing an infection with carprofen administration was assessed in separate animals at day 9 using a screw contaminated with $10^{2}$ CFU S. epidermidis. Quantitative bacteriological analysis assessed bacterial load at euthanasia.

In the $28 \mathrm{~d}$ antibiotic treatment study, carprofen reduced osteolysis but markedly diminished reparative bone formation, although total bacterial load was not affected at euthanasia. Antibiotic efficacy was negatively affected by carprofen (carprofen: 8 out of 8 infected; control: 2 out of 9 infected). Finally, carprofen increased bacterial load and diminished bone formation following reduced S. epidermidis inoculum (10² CFU) at day 9.

This study suggested that NSAIDs with COX-2 selectivity reduced antibiotic efficacy and diminished reparative responses to $S$. epidermidis ODRI.
\end{abstract}

Keywords: Staphylococcus epidermidis, osteomyelitis, in vivo $\mu \mathrm{CT}$, non-steroidal anti-inflammatory drugs, carprofen, antibiotics.

*Address for correspondence: Keith Thompson, AO Research Institute Davos, AO Foundation, Clavadelerstrasse 8, 7270 Davos Platz, Switzerland.

Telephone number: +41 814142325 Email: keith.thompson@aofoundation.org

Copyright policy: This article is distributed in accordance with Creative Commons Attribution Licence (http://creativecommons.org/licenses/by-sa/4.0/).

\begin{tabular}{|c|c|c|c|}
\hline \multirow{5}{*}{ AAALAC } & List of Abbreviations & $\mathrm{BIC}$ & bone-implant contact \\
\hline & & $\mathrm{BR}$ & bone resorption \\
\hline & Association for Assessment and & $\mathrm{BV} / \mathrm{TV}$ & bone fraction \\
\hline & Accreditation of Laboratory Animal & CCL11 & C-C motif chemokine ligand 11 \\
\hline & Care & CFUs & colony-forming units \\
\hline$a g r$ & accessory gene regulator & CoNS & coagulase-negative staphylococcus \\
\hline ANOVA & analysis of variance & COX & cyclooxygenase \\
\hline $\mathrm{BF}$ & bone formation & $\mathrm{CPP}$ & Clinical Priority Program \\
\hline
\end{tabular}




\begin{tabular}{|c|c|}
\hline EDTA & ethylenediaminetetraacetic acid \\
\hline EGF & epidermal growth factor \\
\hline FRI & fracture-related infection \\
\hline G-CSF & $\begin{array}{l}\text { granulocyte colony-stimulating } \\
\text { factor }\end{array}$ \\
\hline GM-CSF & $\begin{array}{l}\text { granulocyte-macrophage colony- } \\
\text { stimulating factor }\end{array}$ \\
\hline GRO & growth-related oncogene \\
\hline IFN- $\gamma$ & interferon gamma \\
\hline IL & interleukin \\
\hline IP-10 & IFN- $\gamma$-induced protein 10 \\
\hline $\mathrm{KC}$ & keratinocytes-derived chemokine \\
\hline LIX & $\begin{array}{l}\text { lipopolysaccharide-inducible CXC } \\
\text { chemokine }\end{array}$ \\
\hline MCP-1 & monocyte chemoattractant protein- 1 \\
\hline MIC & minimum inhibitory concentration \\
\hline MIP & macrophage inflammatory protein \\
\hline MMA & methylmethacrylate \\
\hline NSAID & $\begin{array}{l}\text { non-steroidal anti-inflammatory } \\
\text { drug }\end{array}$ \\
\hline ODRI & orthopaedic-device-related infection \\
\hline PBS & phosphate-buffered saline \\
\hline PEEK & polyetheretherketone \\
\hline PJI & periprosthetic joint infection \\
\hline RANTES & $\begin{array}{l}\text { regulated upon activation, normal } \\
\mathrm{T} \text { cell expressed and secreted }\end{array}$ \\
\hline RAPD & $\begin{array}{l}\text { random amplification of polymorphic } \\
\text { DNA }\end{array}$ \\
\hline ROI & region of interest \\
\hline S. aureus & Staphylococcus aureus \\
\hline S. epidermidis & Staphylococcus epidermidis \\
\hline SD & standard deviation \\
\hline SEM & standard error of the mean \\
\hline SPF & specific-pathogen free \\
\hline TNF- $\alpha$ & tumour necrosis factor alpha \\
\hline TSA & tryptic soy agar \\
\hline TSB & tryptic soy broth \\
\hline VEGF & vascular endothelial growth factor \\
\hline$\mu \mathrm{CT}$ & micro-computed tomography \\
\hline
\end{tabular}

\section{Introduction}

NSAIDs are a drug class that are widely used in human clinical medicine due to their efficacy for reducing inflammation and pain (Jeffcoach et al., 2014; Ong et al., 2007). As inhibitors of the enzyme COX, which exists as a constitutive form (COX1) and an inducible form (COX-2), these drugs reduce prostaglandin synthesis, thereby reducing systemic inflammation and pain. However, since inflammation and prostaglandin E2 play important roles in bone healing (Blackwell et al., 2010; Einhorn and Gerstenfeld, 2015), NSAIDs may have a negative influence if taken during the early inflammationdriven phase of bone healing (Gerstenfeld and Einhorn, 2004; Zura et al., 2016). The impact of NSAIDs on bone healing is a contentious topic in the literature, with a recent systematic review showing no clear trend toward either a positive or negative effect of NSAIDs on bone healing, but highlighting the paucity of high quality studies on the topic (MarquezLara et al., 2016). This problem is further compounded by the issue that NSAIDs are generally available on an over-the-counter basis in numerous countries and, therefore, may be self-administered by the patient without the knowledge of the responsible physician.

ODRIs, including PJI and FRI, occur with an incidence ranging between less than $1 \%$ [PJI after hip prosthesis (Li et al., 2018)] and up to $30 \%$ [FRI after open fracture (Kortram et al., 2017; Puetzler et al., 2019)]. An ODRI is a potentially devastating complication of orthopaedic surgery, both in terms of morbidity and mortality, as well as a financial burden for healthcare systems worldwide (Poultsides et al., 2010). Although the majority of acute ODRIs are caused by S. aureus, a significant proportion of infections are caused by low virulence microorganisms such as S. epidermidis, a CoNS, which may be responsible for up to $40 \%$ of ODRIs (Depypere et al., 2020; Uckay et al., 2009). These bacteria can develop a biofilm on inert materials and necrotic tissues and usually progress with a subclinical presentation, dramatically complicating a diagnosis.

Bone healing in the presence of an implant relies on a subtle balance of pro- and anti-inflammatory mediators and the coordinated activity of resident bone cells and immune cells to orchestrate the healing process. The presence of a bacterial infection dysregulates this process, with an excess of pro-inflammatory signals driving excessive bone destruction and also, depending on the causative pathogen, potentially driving diminished local BF or stimulating ectopic BF (Croes et al., 2019). Therefore, the inhibition of COX by NSAIDs could serve to decrease inflammation-driven bone destruction. However, given the importance of prostaglandins in the bone healing process (Gerstenfeld and Einhorn, 2004; Kaneko et al., 2007; Kawaguchi et al., 1995), administration of NSAIDs may also inhibit reparative responses following infection. The issue is further complicated by the varying selectivity of different NSAIDs for inhibiting different isoforms of COX, with drugs such as ketoprofen displaying COX-1 selectivity, ibuprofen being relatively non-specific and celecoxib being COX-2 selective (Cryer and Feldman, 1998; Mandell, 1999).

In a paper aiming at investigating anti-virulence properties of diflunisal (a COX-1 selective NSAID) on S. aureus, Hendrix et al. (2016) demonstrated that diflunisal protected from osteolysis in a rodent model of S. aureus-induced osteomyelitis. Similar protective effects were also observed with aspirin (also a COX-1 selective NSAID) in a murine model of ODRI (Jiang et al., 2019), thereby suggesting that COX inhibition may prevent infection-induced bone destruction. However, clinical evidence on the effect of NSAID use on fracture healing is limited, with relatively few high-quality studies available. Of particular interest, Jeffcoach et al. (2014), in a retrospective analysis of 1,901 patients with operative fixation of a long-bone fracture, demonstrated that perioperative NSAID use 
Table 1. Overview of study design. $\mathrm{ABs}=$ antibiotics (rifampicin and cefazolin); $\mathrm{QB}=$ quantitative bacteriology.

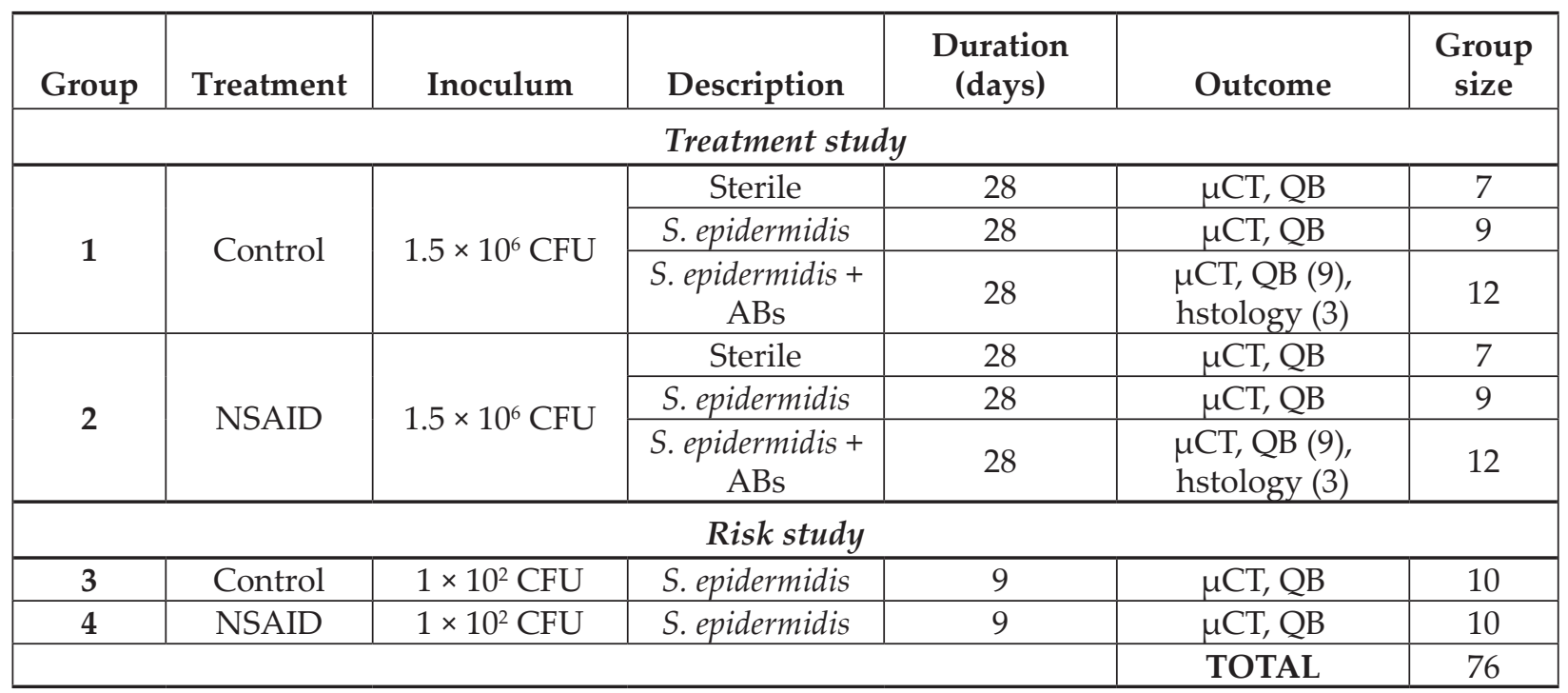

doubles the rate of complications (non-union or FRI), although the data were not subjected to sub-group analysis based on COX selectivity. In a separate study, the development of non-union after nailing of femoral diaphyseal fractures was correlated with NSAID use (Giannoudis et al., 2000). Once again, no discrimination was made concerning COX-1 vs. COX-2 selectivity. A recent retrospective analysis of a large, long-bone fracture cohort provides the only large-scale study comparing NSAID use based on COX selectivity. George et al. (2020) concluded that COX-2 inhibitors, but not non-selective NSAIDs, are correlated with increased rate of non-union. However, whether NSAID treatment directly increases the risk of developing an ODRI or affects the course of an ODRI and/or its response to therapy has not been investigated in a pre-clinical model.

The excellent analgesic potential of NSAIDs underlies their widespread use in current clinical practice, managing pain and inflammation and ultimately permitting the prompt recovery of patients after trauma, but also reducing the necessity for opioid-based medications (of relevance in the context of a global rise of opioid prescription) (Metsemakers, 2020). The current clinical guidelines of many orthopaedic associations recommend the use of NSAIDs for pain management in the perioperative course of total joint arthroplasty or operative fracture treatment (Fillingham et al., 2020), without mention of COX-selectivity. Of note, the 2018 guidelines of the Orthopedic Trauma Association discuss the potential impact of NSAIDs on fracture healing, underpinning the conflicting results evident in the scientific literature. Nevertheless, the guidelines support the use of NSAIDs regarding their proven analgesic potential (Hsu et al., 2019) and lack of detrimental effects when used for short durations. However, to the best of the authors' knowledge, no clinical guidelines exist concerning the use of NSAIDs in the context of an ODRI.
The first aim of the present study was to investigate if treatment with carprofen, a NSAID with preferential COX-2 selectivity, influenced the nature of an $S$. epidermidis ODRI and the response of the infection to antibiotic treatment, using an established rat model of S. epidermidis-induced ODRI and longitudinal $\mu \mathrm{CT}$ scanning over a $28 \mathrm{~d}$ period (Stadelmann et al., 2020; Thompson et al., 2020). In addition, whether perioperative carprofen administration impacted early post-operative infection was investigated. To evaluate this, a markedly reduced inoculum of S. epidermidis was applied on the implanted screw and bacterial colonisation and bone responses were evaluated after a $9 \mathrm{~d}$ observation period.

\section{Materials and Methods}

\section{Study outline}

Consent to perform the study was granted by the ethical committee of the canton of Graubünden, Switzerland (approval numbers: 05/2015, 10/2017 and 04/2019), and was carried out in an AAALACaccredited research institute. A previously reported model of ODRI in the rat proximal tibia was used (Freitag et al., 2019; Stadelmann et al., 2020; Thompson et al., 2020), utilising custom PEEK screws. A total of 76 rats were included in the study (overview of grouping can be seen in Table 1). At 20-24 weeks of age, PEEK screws, either sterile or contaminated with S. epidermidis, were implanted into the left tibia, as described below. Animals in groups 1 and 2 (treatment study) received contaminated screws with a target inoculum of $1.5 \times 10^{6} \mathrm{CFUs}$, which is sufficient to guarantee infection in all animals, based on past experience (see preparation details below). Post-implantation, tibiae were monitored by in vivo $\mu \mathrm{CT}$ at 7 time points over a $28 \mathrm{~d}$ period. In addition, 2 further groups (groups 3 and 4; risk study) were included, to assess whether NSAID treatment 
affected the risk of developing an infection to PEEK screws contaminated with a reduced $S$. epidermidis inoculum $\left(1 \times 10^{2} \mathrm{CFUs}\right)$ as a model of perioperative contamination. Animals in groups 3 and 4 had $\mu \mathrm{CT}$ scans performed immediately post-operatively and at day 9 (euthanasia).

\section{Implant design and manufacturing}

Custom-made screws (5 mm length, $1.5 \mathrm{~mm}$ diameter) were machined from medical grade PEEK containing $20 \%(\mathrm{w} / \mathrm{w})$ barium sulphate (material supplied by Invibio Biomaterials Ltd.) by RISystem AG, Davos, Switzerland.

\section{Bacterial inoculum preparation}

S. epidermidis (strain 103.1; a clinical isolate from a patient with a chronic ODRI and available from the Culture Collection of Switzerland, strain number CCOS 1152) was recovered from frozen stocks and cultured on TSA (Oxoid, Basel, Switzerland) or in TSB (Oxoid) at $37^{\circ} \mathrm{C}$. The S. epidermidis 103.1 strain is sensitive to both antibiotics used in this study, rifampicin and cefazolin, with MIC values of $0.015 \mathrm{mg} / \mathrm{mL}$ and $<1 \mathrm{mg} / \mathrm{mL}$, respectively.

The bacterial inoculum was introduced to the rats on pre-contaminated screws prepared immediately prior to each surgery. On the day of surgery, $S$. epidermidis overnight cultures were centrifuged $(2,500 \times g$ for $10 \mathrm{~min})$, washed in PBS twice and then adjusted to an optical density of $0.50( \pm 0.01)$ at $600 \mathrm{~nm}$. The threaded portion of the screw was submerged and incubated statically at room temperature for $25 \mathrm{~min}$. Test screws were inoculated in parallel within each series of experiments. A quantitative assessment of S. epidermidis adhesion to the test screw was performed by sonication in PBS ( $3 \mathrm{~min}$ ) followed by serial dilution, plating on $5 \%$ horse blood agar (Oxoid) and incubation overnight at $37^{\circ} \mathrm{C}$. The target inoculum for each screw was $1.5 \times 10^{6} \mathrm{CFUs} / \mathrm{screw}$ (range: $0.9-2 \times 10^{6} \mathrm{CFUs} / \mathrm{screw}$ ). All screws were implanted within $30 \mathrm{~min}$ of preparation.

\section{Animal welfare, observation and euthanasia}

Skeletally mature, adult female, SPF Wistar rats, purchased from Charles River (Germany), were used and housed until skeletal maturity (20-24 weeks). Animal monitoring, care and potential exclusion criteria were as previously described (Freitag et al., 2019; Stadelmann et al., 2020; Thompson et al., 2020). Animals were randomly allocated to their group (Table 1). For the $28 \mathrm{~d}$ antibiotic treatment study (groups 1 and 2), all animals were scanned by $\mu \mathrm{CT}$ immediately following surgery (day 0 - to confirm appropriate positioning of screw) and at 6 further time points post-surgery (see details below). For the perioperative risk study (groups 3 and 4), all animals were scanned immediately following surgery (day 0 ) and at day 9 (euthanasia). Animals were weighed at the same time points as $\mu \mathrm{CT}$ scans were taken. Blood samples (using EDTA as an anticoagulant) were also collected from the lateral tail-vein pre-operatively and on days 9, 20 and 28, prior to $\mu \mathrm{CT}$ scans. Plasma was isolated following centrifugation ( $400 \times g, 5 \mathrm{~min})$ and subsequently stored at $-20{ }^{\circ} \mathrm{C}$ until analysis. On day 28 (groups 1 and 2) or day 9 (groups 3 and 4 ), animals were euthanised using an overdose of pentobarbital through intracardiac injection, under isoflurane anaesthesia.

\section{Surgery, anaesthesia and medication administration} Anaesthesia and surgery were performed as previously described (Freitag et al., 2019; Stadelmann et al., 2020; Thompson et al., 2020). In brief, screw insertion surgery was performed at 20-24 weeks, using the previously described protocol (Stadelmann et al., 2015). The mean weight ( \pm SD) of the animals at surgery was $336.0 \pm 26.4 \mathrm{~g}$. Under isoflurane anaesthesia, a sterile or a contaminated screw (for the control and the infected groups, respectively) was inserted in the proximal tibia $2 \mathrm{~mm}$ distal to the growth plate. NSAID groups received a daily carprofen injection $[5 \mathrm{mg} / \mathrm{kg}$, subcutaneously (s.c.)]. Further subgroups of animals infected with $S$. epidermidis were treated with a combination antibiotic regimen ( $25 \mathrm{mg} / \mathrm{kg}$ rifampicin plus $30 \mathrm{mg} / \mathrm{kg}$ cefazolin, s.c.) twice daily from day 7 after screw implantation, for a period of $14 \mathrm{~d}$, followed by a $7 \mathrm{~d}$ washout period, to prevent false negative results in the quantitative bacteriological analysis. Subcutaneous injections of carprofen and the combination antibiotic regimen (rifampicin and cefazolin) were performed at separate sites in the scruff of the neck, using minimal volumes ( $\leq 100 \mathrm{~mL} /$ injection).

\section{In vivo $\mu \mathrm{CT}$ and image processing}

The evolution of peri-prosthetic bone structure following screw implantation surgery was assessed in groups 1 and 2 using time-lapsed in vivo $\mu \mathrm{CT}$ immediately post-operatively and at 3, 6, 9, 14, 20 and $28 \mathrm{~d}$ as previously described (Stadelmann et al., 2020). Groups 3 and 4 were scanned immediately post-operatively and at day 9 . Briefly, animals were scanned under isoflurane anaesthesia and a $10 \mathrm{~mm}$ long ROI, with a diameter of $25.6 \mathrm{~mm}$ field of view and centred on the implanted screw was scanned at a nominal resolution of $25 \mu \mathrm{m}$. Time-lapse $\mu \mathrm{CT}$ image processing was performed to compute BIC, $\mathrm{BV} / \mathrm{TV}, \mathrm{BF}$ and BR rates in a ROI within $700 \mu \mathrm{m}$ from the screw surface. Periosteal reaction was quantified within the medial periosteal region $2 \mathrm{~mm}$ distal and proximal from the screw head. All image processing and analysis were performed using EasyIPL (Web ref. 1), a high-level library of macros using a scanner software (SCANCO Image Processing Language, IPL and OpenVMS Digital Command Language, DCL).

\section{Bacteriology}

Following euthanasia, the respective tibiae were dissected and the screws and bones collected in separate, sterile containers with sterile PBS. Any soft fibrous tissue overlying the protruding head of the screw was also collected in separate sterile containers 
containing PBS. The number of bacteria adhering to the S. epidermidis-contaminated screws was determined by sonicating the screws for $3 \mathrm{~min}$ and vortex mixing for $10 \mathrm{~s}$, before performing serial dilutions and viable bacteria counts on blood agar. Then, the entire tibia from each animal was mechanically homogenised (Omni Tissue Homogeniser and Hard Tissue Homogenising tips; Omni International, Kennesaw, GA, USA) and the quantity of bacteria associated with bone was similarly quantified by serial dilution and viable bacteria counts on blood agar. Soft tissue samples were processed in the same manner. All agar plates were incubated for $24 \mathrm{~h}$ at $37^{\circ} \mathrm{C}$ and checked for contamination or signs of co-infection. Animals were considered as infected when at least one sample (bone, soft tissue or screw) was culture positive. Identification of S. epidermidis 103.1 in culture-positive samples was performed for at least one colony from each culture positive animal using RAPD PCR (Versalovic et al., 1991) following comparison with the original S. epidermidis 103.1 strain.

\section{Histological processing}

A total of 6 randomly chosen animals [3 infected animals treated with antibiotics (group 1) and 3 infected animals treated with NSAIDs and antibiotics (group 2)] were allocated for histological analysis. Following euthanasia, the lower limb was dissected and the overlying skin removed. Then, all samples were fixed for a minimum of 2 weeks in $70 \%$ methanol. After fixation, samples were dehydrated through an ascending ethanol series (70 \%, $96 \%$, absolute ethanol), with two changes for each step, every $2-5 \mathrm{~d}$. Then, samples were transferred to xylene and finally to MMA (Sigma-Aldrich) for embedding. The polymerised samples were sectioned using a Leica 1600 annular blade saw (Leica Biosystems Nussloch $\mathrm{GmbH}$ ). At least two serial sections in the frontal plane through the centre of the screw were made from each sample. One section was used for Giemsa-eosin staining and the remaining section for Brown and Brenn staining, to detect Gram-positive bacteria.

\section{Histopathological analysis (semi-quantitative)}

The slides were analysed by a certified veterinary pathologist (DN) using a semi-quantitative method (grade 0-5) comparing parameters for implant integration, inflammation and infection in both groups. Due to the limited sample number $(n=3)$, histological analysis was performed to give a general morphological overview of the two compared groups and no statistical analysis was performed.

\section{Histomorphometric analysis (quantitative)}

Additionally, a quantitative histomorphometric method was used to measure implant integration. Using Adobe Photoshop 2020 (including analysis plug-in), the bone area was measured in a sleeve of $300 \mu \mathrm{m}$ around the threaded part of the PEEK screw on each individual digital image, with a calibration of 0.452 pixels $/ \mu \mathrm{m}$. The ROI was defined by using the image of the first control sample animal (an identical ROI was applied for analysis of all other samples) and resulted in a polygon around the implant with an area of $~ 6.6 \mathrm{~mm}^{2}$ (exactly $6,654,197 \mu \mathrm{m}^{2}$ ). Its width was defined by the maximum width of the screw head at its neck $(\sim 2,090 \mu \mathrm{m})$, its height by the length of the screw from the neck to the tapered tip $(\sim 3,443 \mu \mathrm{m})$. The bone area inside the ROI was marked by Photoshop's magic wand tool interactively to avoid marking non-bone areas with similar grey values [settings: point sample, tolerance 50 , continuous area, grey value min (mean): 21.1, grey value max (mean): 154.4].

\section{Cytokine measurements}

Plasma samples collected during the $28 \mathrm{~d}$ study [day 0 (pre-operative), 9, 20 and 28] were analysed for inflammatory cytokines using a 27-plex Milliplex xMAP Rat Cytokine/Chemokine Magnetic Bead Panel (EMD Millipore) according to manufacturer's instructions. Plasma $(25 \mu \mathrm{L})$ was used to analyse the following cytokines/chemokines/growth factors in the Luminex immunoassay: EGF, eotaxin/CCL11, fractalkine, G-CSF, GM-CSF, GRO/KC, IFN- $\gamma$, IL$1 \alpha$, IL-1 $\beta$, IL-2, IL-4, IL-5, IL-6, IL-10, IL-12 (p70), IL-13, IL-17A, IL-18, IP-10, leptin, LIX, MCP-1, MIP- $1 \alpha$, MIP-2, RANTES, TNF- $\alpha$ and VEGF. Based on instrument settings and standard curves, the assay limit of detection was set at $10 \mathrm{pg} / \mathrm{mL}$. Among the 27 proteins measured in the immunoassay, the following cytokines were expressed at undetectable concentrations (<10 pg/mL): G-CSF, GM-CSF, MIP$1 \alpha$, IL-2, EGF and TNF- $\alpha$ and the corresponding data are, therefore, not presented.

\section{Statistical analysis}

$\mu \mathrm{CT}$ time series curves are shown as mean \pm SEM for the measured data points. Two-way ANOVA with a Tukey's post-hoc test for multiple comparisons was used to analyse changes in bone parameters over time and cytokine measurements. Kruskall-Wallis tests were used to analyse quantitative bacteriology data. The Fisher Exact Test was used to check for differences in proportions of infected animals between groups. Threshold for statistical significance was set as $p<0.05$. All analyses were performed using GraphPad Prism software (GraphPad Software, Inc.).

\section{Results}

\section{Animal welfare}

All animals recovered well from surgery and anaesthesia. In groups 1 and 2 (28 d antibiotic treatment study), mean weight loss following surgery was $\sim 4.7 \%$ of initial body weight at $3 \mathrm{~d}$. All animals recovered to pre-operative body weight by $28 \mathrm{~d}$. The recovery of body weight following surgery was not significantly affected by S. epidermidis infection, 
antibiotic treatment or NSAID administration (data not shown). One animal from group 1 (infected + antibiotics) was found dead in the cage during the study and was not replaced. Data pertaining to this animal were excluded from the subsequent analysis.

\section{Bone changes in vicinity of implant}

Rats receiving screws contaminated with S. epidermidis developed marked osteolysis in the vicinity of the screw by day 6, with maximal decreases in BIC and BV/TV observed at day 14 (Fig. 1 and Fig. 2). Consistent with this, there was a significant reduction in BF and a marked increase in BR evident at day 6 (Fig. 3). The osteolytic response to infection was also associated with a subsequent reparative response, which became evident at day 14. Specifically, an extensive periosteal reaction was observed from day 14 onwards, which was also associated with the deposition of further mineralised tissue within the vicinity of the implant (Fig. 1,3).

Daily administration of carprofen to non-infected animals had limited effects on bone parameters. However, carprofen administration resulted in pronounced changes in the response of bone tissue to a S. epidermidis infection. Osteolytic responses in the vicinity of the screw were markedly reduced following carprofen administration, with a trend for increased BV/TV relative to infected control animals observed at day $6(p=0.059)$ and day $9(p=0.070)$ (Fig. $2)$. Of particular note, the rate of BR was significantly diminished at day 6 with carprofen administration $(p=0.021)$. However, the most pronounced effects of carprofen concerned BF, with significantly reduced periosteal reaction on days 9-28 (day 9, $p=0.002$; day 14, $p<0.001$; day 20, $p=0.026$; day 28, $p=0.012$ ) and also a trend for reduced rates of $\mathrm{BF}$ at days 20
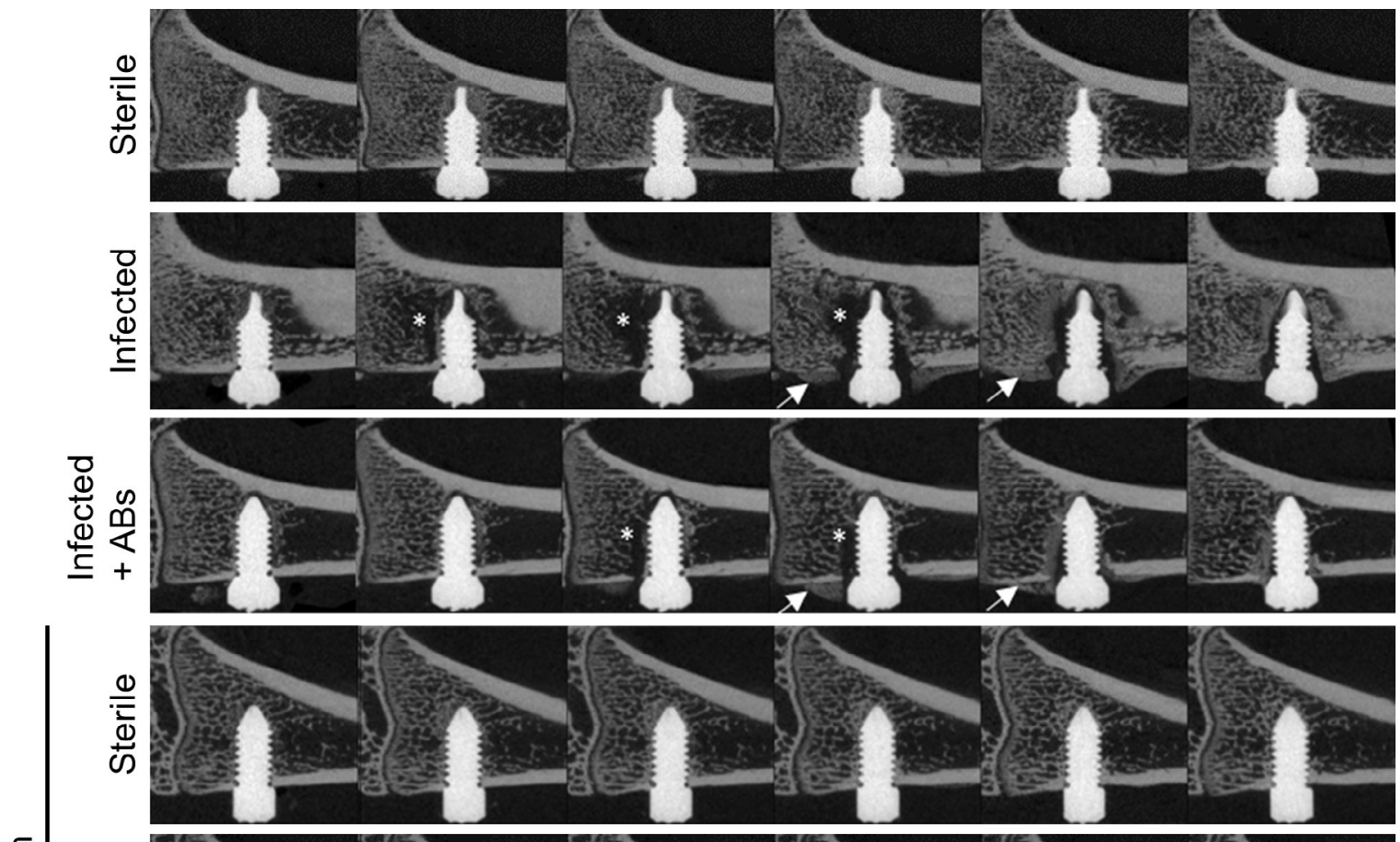

$\frac{1}{0}$
$\frac{0}{0}$
$\frac{2}{2}$
$\frac{2}{0}$
+
+
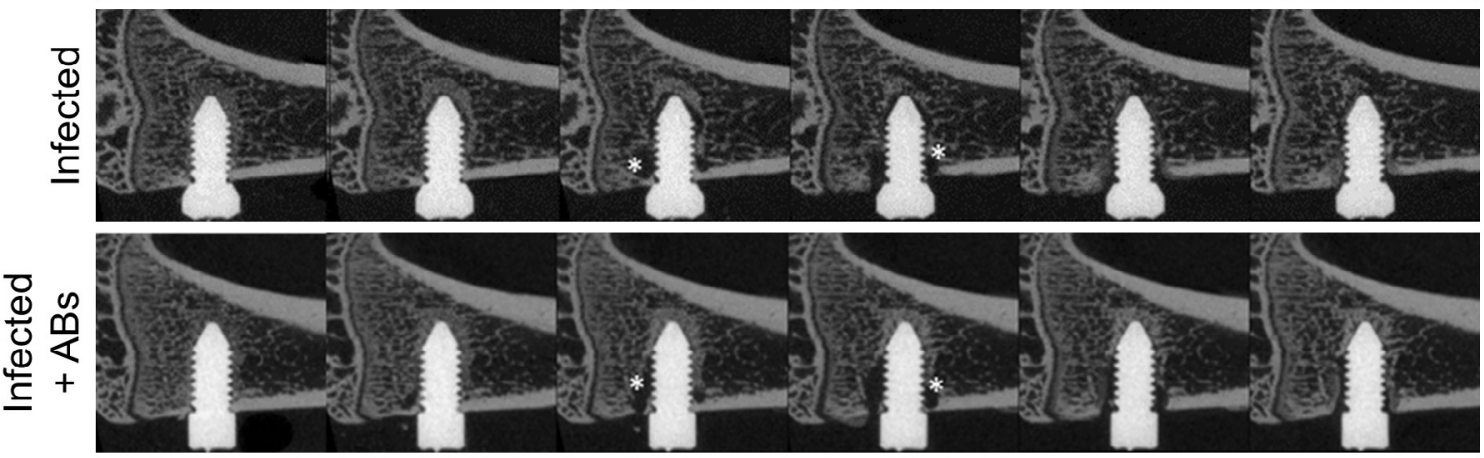

Day 0

6

9

14

20

28

Fig. 1. Representative time series of longitudinal in vivo $\mu \mathrm{CT}$ scans from S. epidermidis-infected animals and following carprofen administration, in comparison to non-infected animals (sterile). The effect of a combination antibiotic regimen ( $\mathrm{ABs}$ ), when administered from days 7-21, is also shown. Representative images are from the same animal and aligned to the baseline (day 0 ) scan, chosen based on median BV/TV values from each experimental group. Asterisks indicate regions of osteolysis and white arrows indicate periosteal BF. Note the limited efficacy of antibiotic treatment for preventing bone changes in response to infection and the reduced osteolytic response and absence of periosteal BF in the carprofen-treated groups. 
$(p=0.061)$ and $28(p=0.108)$ (Fig. 3). No changes in BIC were observed between infected control and NSAID-treated animals.

\section{Bone changes in response to antibiotic therapy}

The efficacy of a combination antibiotic therapy on the bone response to $S$. epidermidis infection was assessed. Administration of rifampicin and cefazolin on day 7 did not significantly affect S. epidermidisinduced changes in $\mathrm{BIC}$ or BV/TV relative to infected animals or infected animals receiving carprofen (Fig. 1 and Fig. 2). Similarly, antibiotic therapy did not change periosteal reaction or rates of $\mathrm{BF}$ or resorption (Fig. 3) in infected animals in the absence or presence of carprofen.

\section{Histomorphometric analysis}

The amount of bone tissue near the implant was assessed in a ROI of $300 \mu \mathrm{m}$ around the threaded part of the PEEK screw. Due to previous observations, whereby antibiotic administration did not alter $S$. epidermidis-induced bone parameters (Fig. 2, 3), and for increased clinical relevance, all animals chosen for this aspect of the study had received antibiotic therapy. In 3 randomly chosen animals (receiving S. epidermidis-inoculated screws and antibiotics), carprofen administration reduced the amount of bone tissue present in the vicinity of the screw by $\sim 38 \%$ compared to control animals (mean bone area \pm SD: control $=1.098 \pm 0.166 \mathrm{~mm}^{2}$; NSAID $\left.=0.680 \pm 0.110 \mathrm{~mm}^{2}\right)$ (Fig. 4a). This reduction in bone tissue was more pronounced in the medullary region compared to the cortical region, which was consistent with $\mu \mathrm{CT}$-based observations.

\section{Histopathological analysis}

None of the infected control or carprofen-treated animals that received antibiotic therapy displayed evidence of focal or diffuse inflammation in the bone marrow (i.e. no myelitis with/without formation of micro-abscesses), but did exhibit a low grade, diffuse activation, predominantly involving the myelopoietic lineage. Limited evidence of Giemsa-positive coccoid bacteria was observed in the Giemsa-eosin-stained
Bone-implant contact

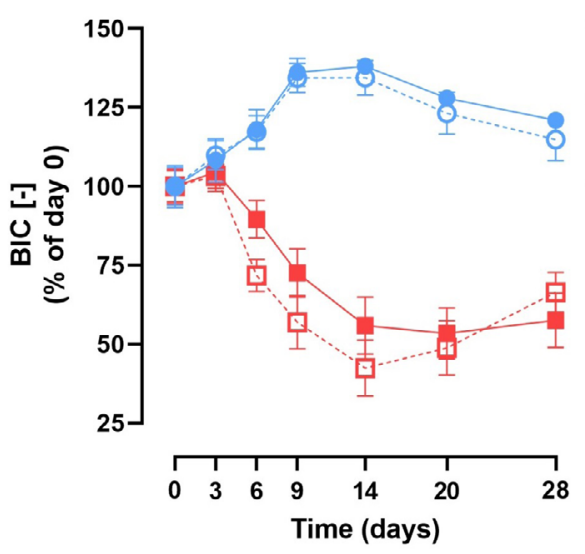

Bone-implant contact

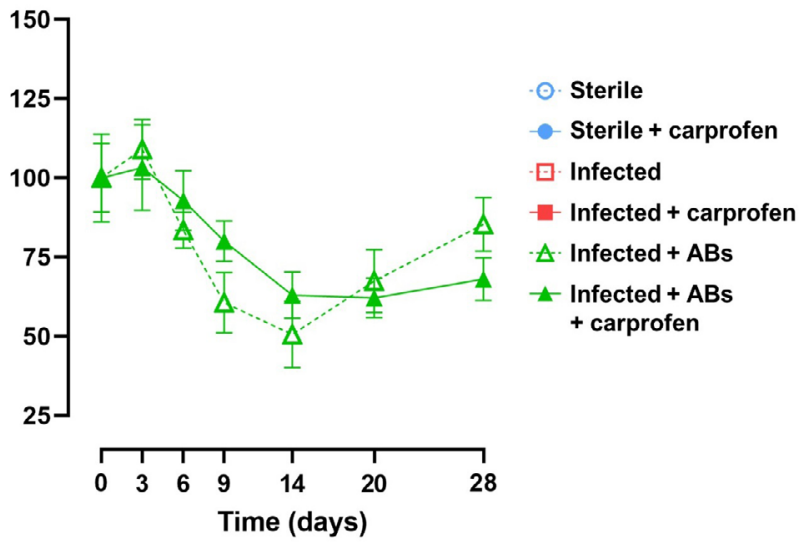

Bone fraction

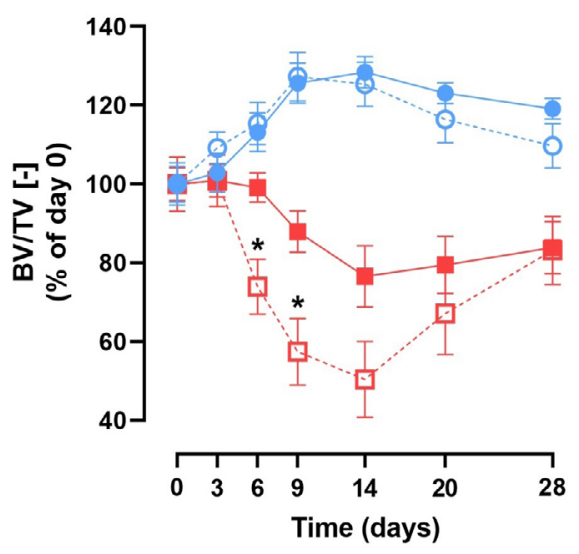

Bone fraction

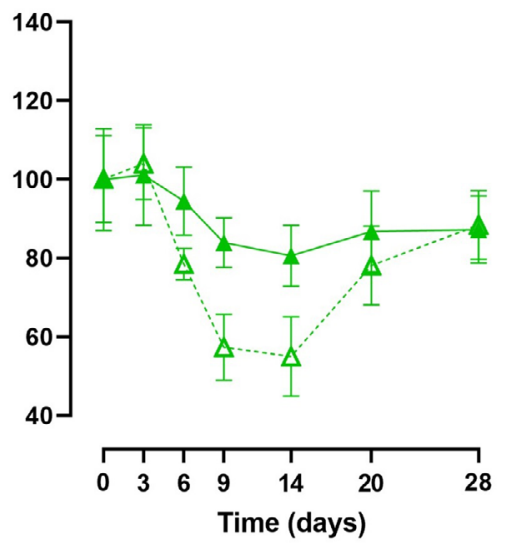

Fig. 2. S. epidermidis infection led to changes in normalised BIC (top row) and BV/TV (bottom row), over time. Responses in non-infected animals (sterile), infected animals and impact of carprofen administration are shown in the left panels. The influence of antibiotic therapy on bone changes in the absence or presence of carprofen is shown in the right panels. Data shown are the mean \pm SEM. Two-way ANOVA with a Tukey's post-hoc test for multiple comparisons was performed to assess significant differences between non-carprofenand carprofen-treated infected animals, in the absence or presence of antibiotic therapy. ${ }^{*} p<0.05$. 
Periosteal reaction

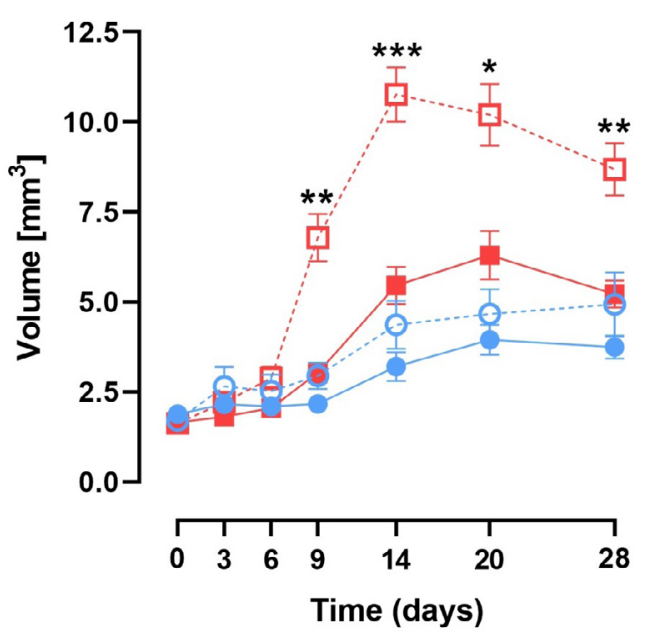

Bone formation

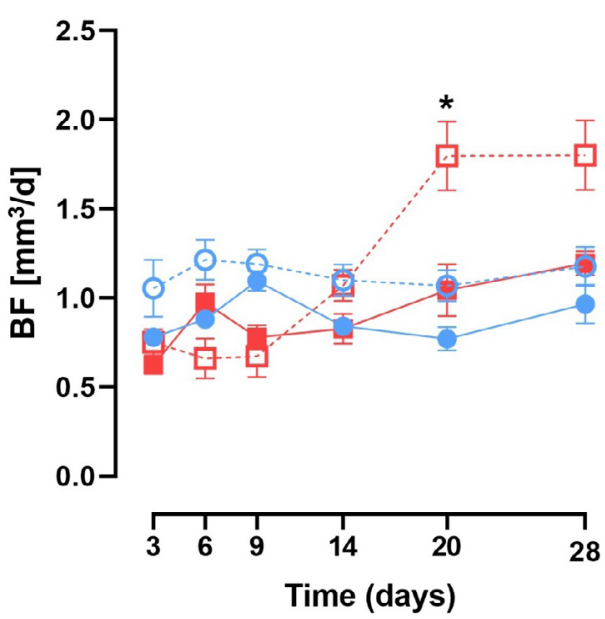

Bone resorption

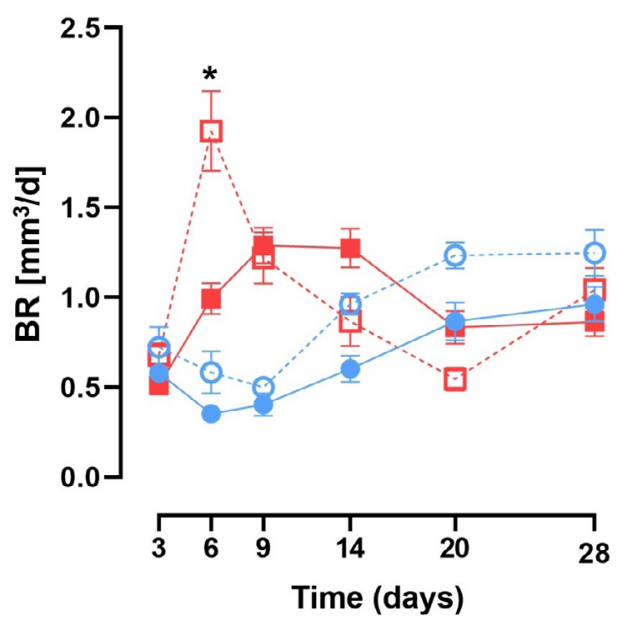

Periosteal reaction

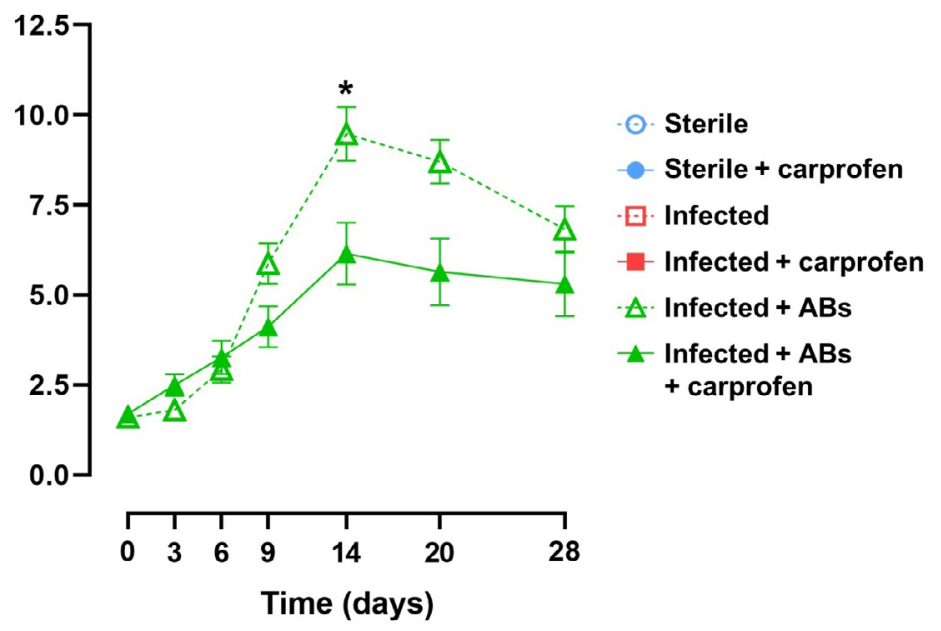

Bone formation

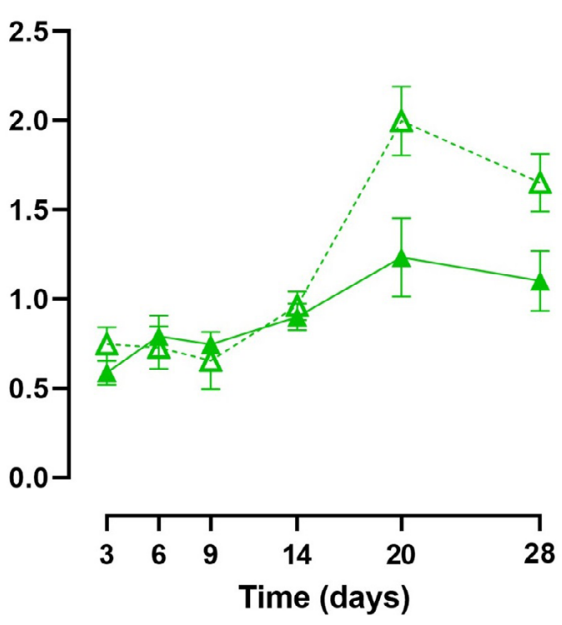

Bone resorption

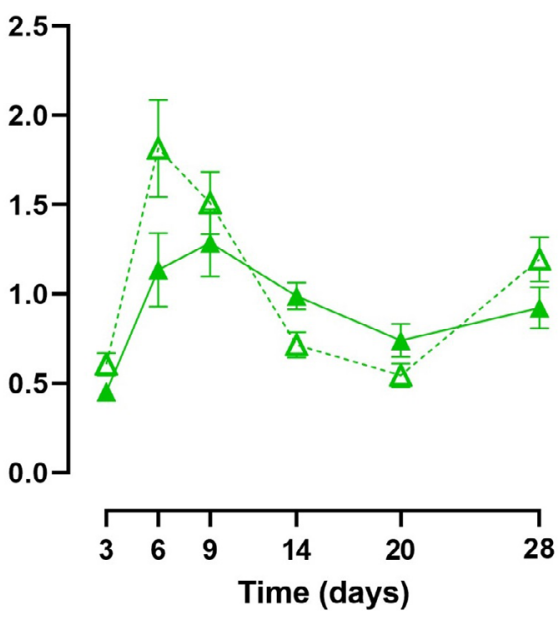

Fig. 3. S. epidermidis infection led to changes in periosteal BF (top row) as well as rates of BF (middle row) and BR (bottom row) over time. Responses in non-infected animals (sterile), infected animals and the impact of carprofen administration on each parameter are shown in the left panels. The influence of antibiotic treatment in the absence or presence of carprofen is shown in the right panels. Data shown are the mean \pm SEM. Two-way ANOVA with a Tukey's post-hoc test for multiple comparisons was performed to assess significant differences between non-carprofen- and carprofen-treated infected animals, in the absence or presence of antibiotic therapy. ${ }^{*} p<0.05,{ }^{* *} p<0.01,{ }^{* * *} p<0.001$. 
section, which was also confirmed by BrownBrenn staining (Fig. 4b), in 1 out of 3 animals in the carprofen-treated group, whereas no bacteria were detected in the control animals. These bacteria were found on sequestered and necrotic bone particles near the implant thread (data not shown).

\section{Effect of carprofen on bacterial burden}

The viable bacteria resident in the proximity of the implanted screw were quantified to determine the impact of carprofen administration and the efficacy of the antibiotic regimen. All S. epidermidis-infected animals that were not treated with antibiotics remained infected at euthanasia. In addition, all animals receiving sterile screws were culture- negative at euthanasia (data not shown). Despite the decreased osteolysis evident with carprofen administration, there was no difference between mean CFU counts in the infected animals as compared to the NSAID-treated infected animals (mean CFU count \pm SEM: control $=53,686 \pm 22,698$ CFUs; NSAID $=45,617 \pm 12,019$ CFUs, $p>0.999$ ) (Fig. 5). Furthermore, whilst the antibiotic therapy had no detectable effect on any infection-induced changes in the assessed bone parameters, the antibiotic regimen was markedly effective at reducing bacterial burden in infected animals (mean CFU count $=2$ '506 $\pm 1,776$; $p=0.016)$, with the successful clearance of the infection in 7 out of 9 animals. However, antibiotic efficacy was dramatically reduced in carprofen-

a
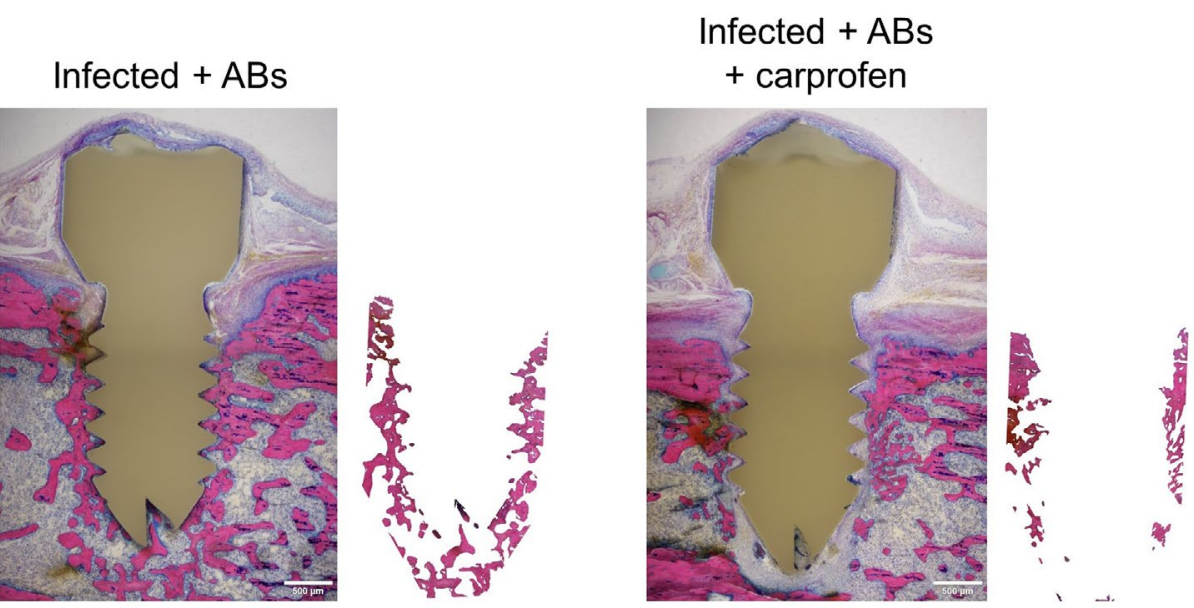

b
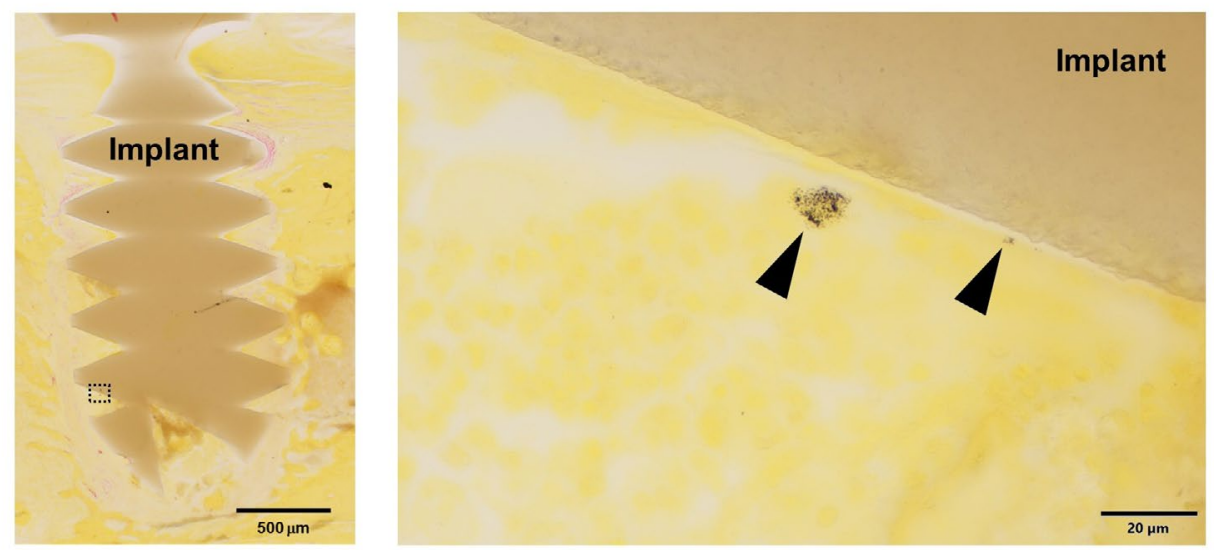

Fig. 4. Histological and histopathological assessment of bone changes resulting from implantation of S. epidermidis contaminated screws in the absence or presence of carprofen administration after $28 \mathrm{~d}$. MMA-embedded thick sections, cut in the frontal plane through the centre of the PEEK implant, were stained with (a) Giemsa-eosin, and the proportion of trabecular bone resident in a defined ROI was then quantified, or (b) Brown-Brenn stain to detect the presence of bacteria. (a) Representative images of the implanted screw, with the bone content of the selected ROI used for quantification shown to the right of the overview image, in the absence (left) or presence (right) of carprofen. Magnification: $4 \times$ objective; scale bar $=500 \mu \mathrm{m}$. (b) Left panel: overview image from 1 carprofen-treated animal showing the presence of the implant and highlighting the region of the higher magnification view in the right panel (dashed box). Magnification: $4 \times$ objective; scale bar $=500 \mu \mathrm{m}$. Right panel: higher magnification view (dashed box in the left panel) displaying evidence of S. epidermidis microcolonies (indicated by black triangles) in the vicinity of the implanted screw. Magnification: 100× objective (oil); scale bar $=20 \mu \mathrm{m}$. 
treated animals, with only a minor reduction in the mean CFU count $(16,438 \pm 6,426)$ and a complete failure to clear the infection in all (8 out of 8 ) animals $(p=0.002$, Fisher's exact test). Bacterial colonies taken from animals found to be culture-positive despite antibiotic therapy were also assessed for resistance to rifampicin but were found to be equally susceptible to rifampicin as the parental strain (data not shown).

\section{Effect of carprofen on inflammatory responses due} to $S$. epidermidis infection

Whether the differences in bacterial CFU counts after antibiotic therapy were due to disparities in inflammatory responses induced by carprofen administration was examined. Plasma samples collected over time were analysed for cytokine/ chemokine secretion using multiplex immunoassay. Trending upregulation of proinflammatory cytokines such as IL-6, IFN- $\gamma$, IL-12p70 and MCP-1 was observed in all rats at day 9 post-inoculation (Fig. 6). However, no statistically significant differences between the carprofen-treated and the control group were found. All other cytokines/chemokines

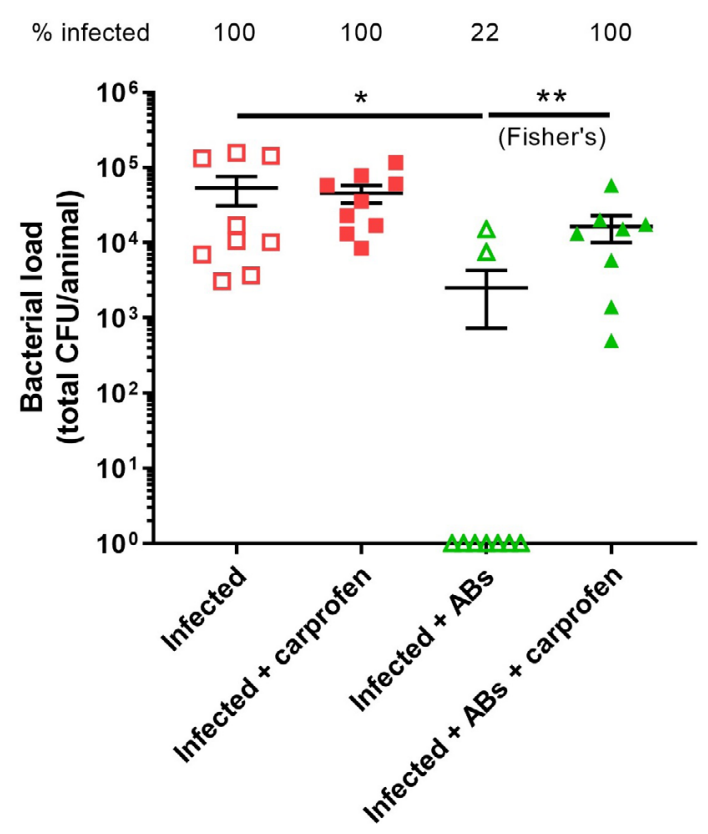

Fig. 5. Quantitative bacteriological assessment indicating total bacterial load per animal and the percentage of infected animals per group after $28 \mathrm{~d}$. Culture-negative samples were arbitrarily assigned a value of 1 CFU to permit display on a logarithmic axis. Data shown are the total CFU counts from each animal with mean \pm SEM indicated. 9 animals were included per group, with the exception of the infected $+\mathrm{ABs}+$ carprofen group, where 1 animal was excluded due to the detection of a polymicrobial infection following dissection. A Kruskall-Wallis test with Dunn's post-hoc test for multiple comparisons was performed to compare differences in CFU counts between groups. ${ }^{*} p<0.05$. A Fisher's exact test (indicated) was performed to compare proportions of infected animals between antibiotic-treated groups in the absence or presence of carprofen. ${ }^{* *} p<0.01$. measured showed no significant differences between groups (data not shown).

Impact of carprofen administration on risk of developing an infection

Following the characterisation of the bone changes and inflammatory responses induced by the standard inoculum of $S$. epidermidis on the implanted screw $\left(\sim 1.5 \times 10^{6}\right.$ CFUs $)$, it was determined whether carprofen administration would influence the risk of developing, or the severity of, an infection following implantation of a screw inoculated with a markedly reduced amount of $S$. epidermidis $\left(1 \times 10^{2}\right.$ CFUs $)$ within the early post-operative period. Most control animals (9 out of 10) and all carprofen-treated animals (10 out of 10), receiving the reduced inoculum of $S$. epidermidis (i.e. 4 orders of magnitude less than the standard CFU inoculum) were infected at day 9 (Fig. $7)$. Interestingly, in contrast to earlier findings at day 28 , carprofen administration significantly increased mean bacterial load by 16-fold over control animals at day 9 post-inoculation (mean CFU count \pm SEM: control $=3,030 \pm 2,491 ;$ NSAID $=47,495 \pm 37,731$; $p=0.018$ ).

The next step was to characterise the bone changes occurring in response to implantation of a screw with the low S. epidermidis inoculum and the impact of carprofen administration on these responses at day 9. Conversely to the findings with the standard inoculum, $1 \times 10^{2}$ CFUs $S$. epidermidis did not decrease $\mathrm{BIC}$ or $\mathrm{BV} / \mathrm{TV}$ values, with an overall trend for increased $\mathrm{BF}$ in control animals at $9 \mathrm{~d}$ (Fig. 8). Of particular note, despite these observed differences in bone responses between the low and standard S. epidermidis inoculum, $1 \times 10^{2} \mathrm{CFUs} S$. epidermidis induced a comparable periosteal reaction volume to that observed with the standard inoculum at $9 \mathrm{~d}$ (Fig. $3,8)$. Carprofen administration did not significantly affect bone responses in terms of BIC, BV/TV or rates of bone formation/resorption at $9 \mathrm{~d}$, compared to control animals. However, carprofen administration significantly reduced periosteal reaction at $9 \mathrm{~d}$ (Fig. 8).

\section{Discussion}

Pro-inflammatory cytokine production in response to ODRI stimulates bone destruction, which may be diminished by anti-inflammatory drugs such as NSAIDs. However, since NSAIDs inhibit the production of prostaglandins, which are important mediators of the bone healing cascade, administration of NSAIDs may also be associated with inhibitory effects on reparative responses of bone following injury or infection. The findings of the present study indicated that administration of carprofen, a preferential COX-2 selective NSAID, reduced $S$. epidermidis-induced osteolysis but also markedly reduced reparative bone-forming responses. In addition, carprofen exacerbated bacterial burden at 
a

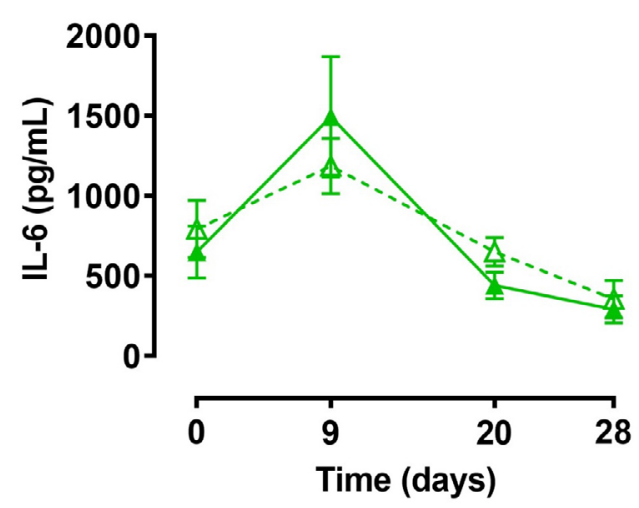

C

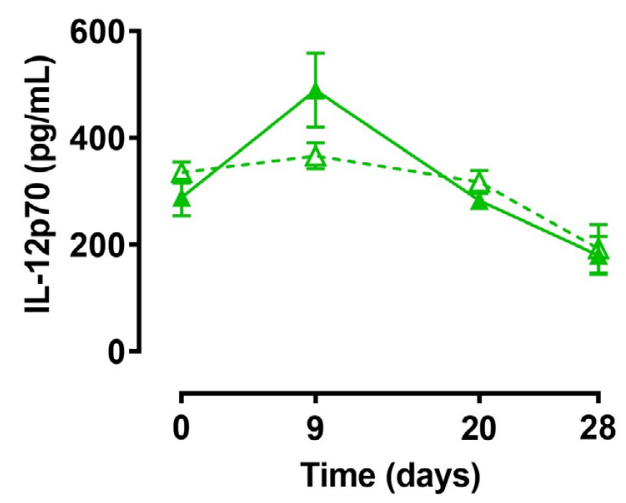

b

$\triangle \cdot$ Infected + ABs

- Infected + ABs + carprofen

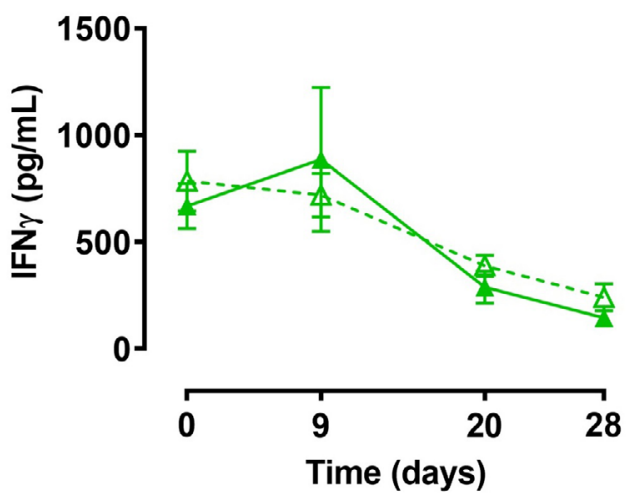

d

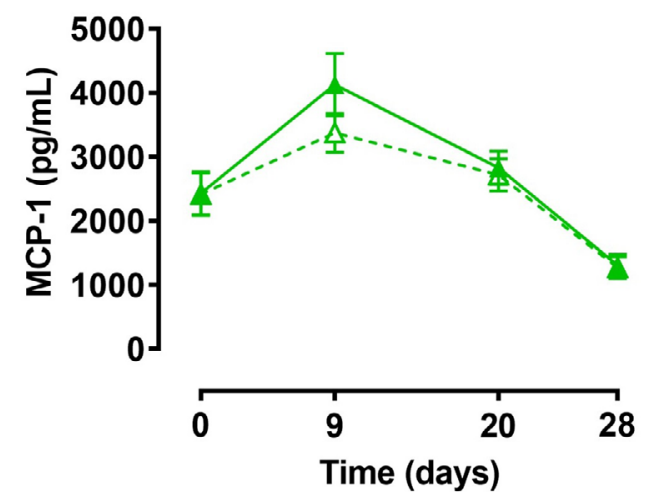

Fig. 6. Influence of carprofen administration on inflammatory cytokine secretion in S. epidermidis infected animals. Plasma samples collected over time [day 0 (pre-operatively), 9, 20, 28] from infected animals treated with antibiotics, with or without carprofen, were subjected to inflammatory cytokine secretion analyses using a bead-based multiplexed Luminex immunoassay ( $n=11-12$ animals in each cohort).

\% infected $\quad 90 \quad 100$

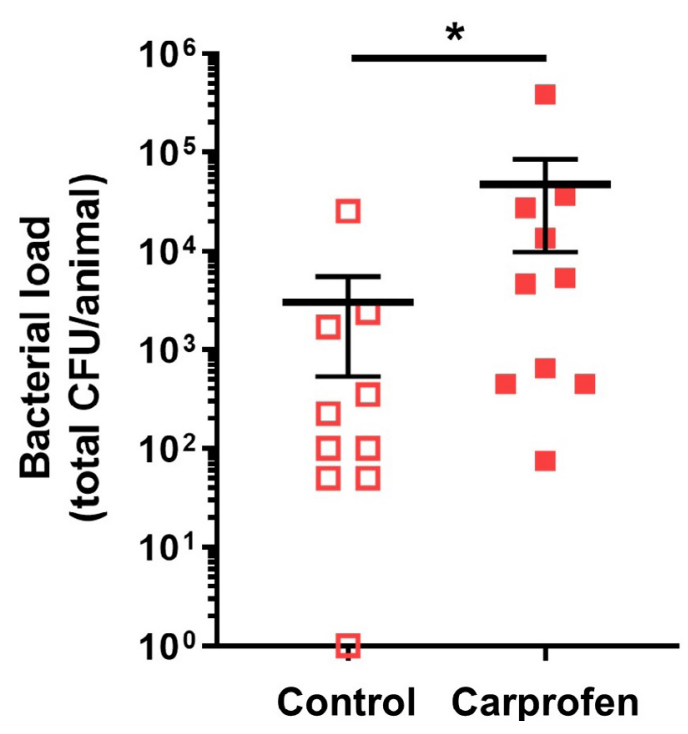

Fig. 7. Quantitative bacteriological assessment of total bacterial load per animal after $9 \mathrm{~d}$ following implantation of a screw contaminated with $10^{2} \mathrm{CFU}$ of S. epidermidis. Culture-negative samples were arbitrarily assigned a value of 1 CFU to permit display on a logarithmic axis. Data shown are the total CFU counts from individual animals with mean \pm SEM indicated. 10 animals were included per group. A Mann-Whitney test was performed to compare differences in CFU counts between groups. ${ }^{*} p<0.05$. 
an early phase of the infection and negatively affected antibiotic efficacy for clearing S. epidermidis infections in the presence of an implant.

Using an established $\mu \mathrm{CT}$-based model for monitoring bone infection, complex effects of carprofen were observed on the response of bone to $S$. epidermidis infection. Perhaps the most striking finding concerned the decrease in antibiotic efficacy in carprofen-treated infected animals. This is consistent with a previous study reporting that non-selective NSAIDs decrease antibiotic efficacy in group-A streptococci-induced necrotising fasciitis in mice (Hamilton et al., 2014). Although the specific mechanism for such an interaction of NSAIDs with antibiotics is currently unknown, NSAID administration may be contributing to bacterial survival in the presence of antibiotics by increasing multidrug efflux pump expression in bacteria and/or decreasing expression of antibiotic target proteins, as reported for the active metabolite of aspirin, salicylate (Hartog et al., 2010; Riordan et al., 2007; Shen et al., 2011; Zimmermann and Curtis, 2018). Conversely, it is purported that some NSAIDs could be substrates for the efflux pump in Gram-negative rods (Laudy et al., 2016) and also in S. aureus (Price et al., 2002), leading to saturation of the efflux pump, thereby increasing the intracellular antibiotic concentration and suggesting a synergistic effect of NSAIDs with antibiotics. Other reported examples of potential synergistic interactions of NSAIDs with antibiotics includes diclofenac, which may act indirectly by increasing the pharmacodynamics of ciprofloxacin when both are co-administered (Iqbal et al., 2009), and celecoxib, which may sensitise $S$. aureus to antibiotics by altering membrane potential and increasing membrane permeability (Annamanedi and Kalle, 2014; Varma et al., 2019). Plasma cytokine data suggested that carprofen administration did not seem to influence systemic pro-inflammatory responses due to $S$. epidermidis infection up to $28 \mathrm{~d}$ post-infection. However, it cannot be discounted that long-term NSAID administration may impair the host immune response to $S$. epidermidis infection, thereby allowing persistence of the infection despite antibiotic

\section{Bone fraction}

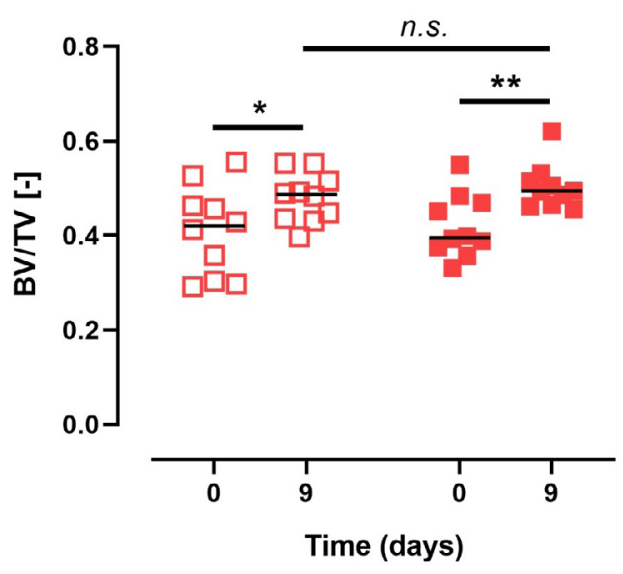

Bone formation

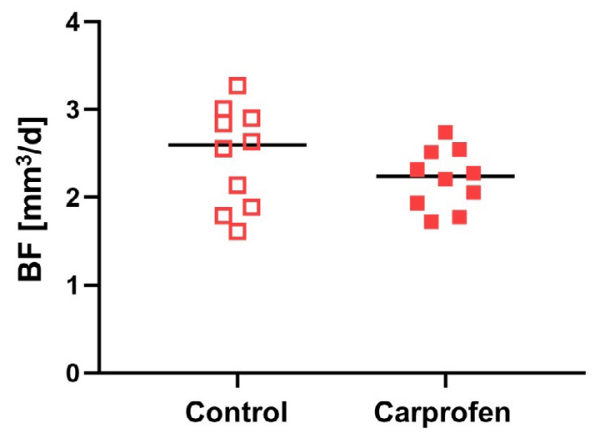

Periosteal reaction

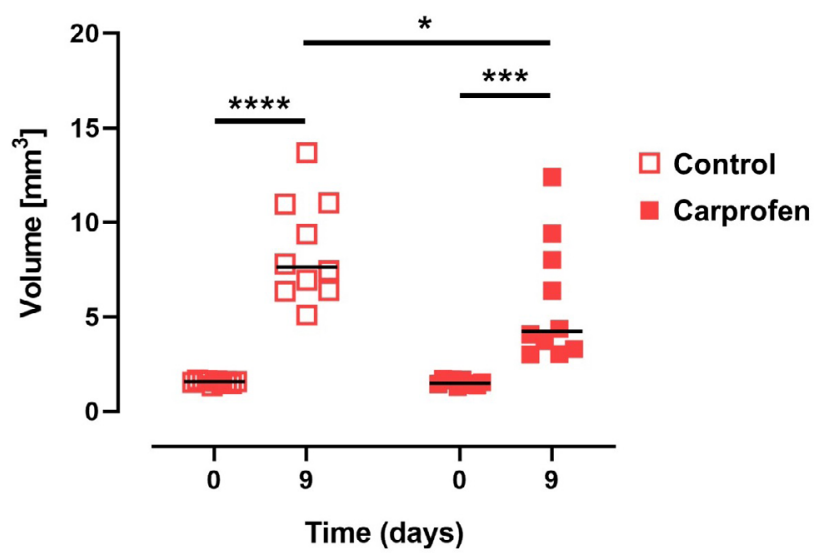

Bone resorption

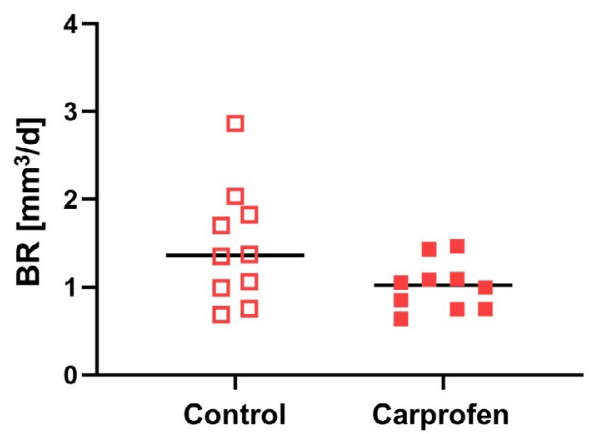

Fig. 8. Bone changes induced by a low inoculum of S. epidermidis in the absence (Control) or presence of carprofen administration (Carprofen) after $9 \mathrm{~d}$. Bone fraction (BV/TV; top row, left panel), periosteal reaction volume (top raw, right panel) and rates of new bone formation (bottom row, left panel) and bone resorption (bottom row, right panel) are shown. Data shown are from individual animals with the mean indicated by the horizontal bar. One way-ANOVA with Tukey's post-hoc test was performed to determine significant differences between $>2$ groups. Paired $t$-tests were performed to assess changes in rates of BF and resorption at 9 d. n.s.: not significant. ${ }^{*} p<0.05,{ }^{* *} p<0.01$, ${ }^{* * *} p<0.001,{ }^{* * * *} p<0.0001$. 
treatment. Nevertheless, given the findings of the study, chronic NSAID administration in combination with antibiotic therapy is not without risk and, therefore, should be carefully considered. Further studies are necessary to elucidate the underlying mechanism for this phenomenon, given the potential for complex pharmacokinetic and pharmacodynamic interactions with such combination drug regimens.

Carprofen displayed limited effects on osseointegration of the non-inoculated implant. However, carprofen administration in the presence of $S$. epidermidis resulted in diminished osteolysis in the vicinity of the implant. This observation was consistent with the bone protective effects of NSAID treatment previously observed in rodent models of $S$. aureus infection in the absence of an implant, including the non-selective NSAID ibuprofen (Rissing and Buxton, 1986) and the COX-1 selective NSAID diflunisal (Ford et al., 2020; Hendrix et al., 2016), which was interestingly shown to exhibit anti-virulence properties unrelated to its inhibitory effects on COX. Although the underlying mechanism for these bone protective effects in S. epidermidis osteomyelitis is currently unknown, previous studies have identified that diflunisal inhibits the quorumsensing agr pathway in S. aureus, thereby limiting virulence factor production (Khodaverdian et al., 2013) and protecting osteoblasts from cell death (Hendrix et al., 2016). Since S. epidermidis lacks the cytolytic virulence factors of $S$. aureus, the bone protective effect of NSAID administration that was observed suggested that prostaglandin E2 is likely generated in response to $S$. epidermidis infection and drives osteoclast-mediated bone destruction, since prostaglandin E2 is a known stimulator of osteoclast formation (Kaneko et al., 2007). However, longitudinal $\mu \mathrm{CT}$ analysis also revealed profound changes in the capacity of new bone to form (defined as deposition of periosteal woven bone and medullary cancellous BF) following infection in animals treated with carprofen. Such inhibitory effects of NSAIDs (both selective and non-selective) on bone repair following stress fracture have previously been reported in rodent models (Kidd et al., 2013) and in human patients (Hughes et al., 2019). As far as it can be ascertained, this is the first demonstration that a NSAID inhibited reparative responses to bone infection with a low virulence organism such as S. epidermidis in the presence of an implant. The pronounced periosteal-derived BF in control animals in response to $S$. epidermidis infection was consistent with previous reports demonstrating that the presence of either live or dead S. aureus bacteria on an implant generates both osteolysis and new BF (Croes et al., 2017; Croes et al., 2019). The present study data indicated that such inflammationinduced $\mathrm{BF}$ in response to bacterial infection was sensitive to carprofen, suggesting that prostaglandin E2 may play a role in the reparative bone-forming response to bacterial antigens.

The failure of carprofen to affect osseointegration (as assessed using BIC measurements as a proxy) of a sterile implant was in contrast to other reports. Trancik et al. (1989) described a reduction in bone ingrowth of porous coated cobalt-chrome implants in rabbits after treatment with the non-selective NSAIDs indomethacin or ibuprofen; Cook et al. (1995) reported retarded osseointegration of porous-coated titanium implants in an indomethacin-treated canine model at early time-points (3 weeks), although this difference was not observed at later time-points (24 weeks). Studies from clinical dentistry have also suggested a negative effect of NSAIDs on implant osseointegration (Etikala et al., 2019; Winnett et al., 2016). Interestingly, some authors have suggested a detrimental effect in the case of selective COX-2 inhibition (Gomes et al., 2015). Indeed, a model of COX-2-deficient mice showed dramatically reduced osseointegration of dental implant (Chikazu et al., 2007). Clinical data in orthopaedic surgery offer conflicting results, perhaps reflecting the variability of COX-isoform selectivity of the evaluated NSAIDs. A prospective study on 80 patients receiving indomethacin (which displays COX-1 selectivity) for preventing heterotopic ossification after total hip arthroplasty did not reveal any trend for decreased osseointegration or increased implant-loosening after a 6 year follow-up (Wurnig et al., 1999). In contrast, a randomised trial on total hip arthroplasty patients receiving ibuprofen for prophylaxis of heterotopic ossification revealed a significantly higher risk of revision for aseptic loosening at 10 years in the NSAID-treated group (Persson et al., 2005). The specific NSAID used in the present study, carprofen, is a reversible COX inhibitor, with a preference for COX-2 in a variety of different species (Brideau et al., 2001; Radi, 2009), although this COX-2 selectivity is markedly lower than that of highly COX-2 selective drugs such as celecoxib. Thus, specific NSAIDs with differing selectivity for COX-1 vs. COX-2 may be associated with increased risk of aseptic loosening, although further large-scale studies are necessary to clarify this issue.

While the study findings demonstrated that carprofen administration did not increase bacterial load at late time points in response to infection which was consistent with an earlier study by Rissing and Buxton (1986) - an increased bacterial load was observed at the point of peak osteolysis (day 9) when administering a markedly reduced inoculum of $S$. epidermidis. Although these low inoculum studies were designed to determine whether carprofen treatment promoted the risk of developing an implant-related infection, it was found that even $10^{2}$ CFUs of S. epidermidis (i.e 4 orders of magnitude less than used in the routine model) could reliably establish an infection in $\geq 90 \%$ of experimental animals. This lower inoculum was also more realistic in terms of the number of bacteria that may enter a wound in the early peri-operative or post-operative period. This highlights the fundamental importance of an implant for establishing an infection where profoundly reduced inoculum doses can reliably 
trigger an infection in host animals, as previously reported by Zimmerli et al. (1982): $10^{2}$ CFUs of $S$. aureus could establish an infection in $95 \%$ of implants in a preclinical guinea pig model, while $10^{8}$ CFUs were unable to form abscesses in the absence of an implant. However, such potential effects of NSAIDs for exacerbating bacterial load in ODRI patients are poorly understood. Although some authors have suggested a relationship between NSAID administration and more severe course of bacterial infection, no clinical trial to date has confirmed this. No increased risk for septic shock was reported in NSAID-treated bacteriemia patients in a multi-centre case control study (Legras et al., 2009). More recently, a randomised double blind clinical trial could not establish a link between aspirin (a relatively COX-1 selective NSAID) and death associated with sepsis (Eisen et al., 2020).

A specific limitation of the study concerned the chronic nature of the NSAID dosing regimen administered. However, NSAID administration is typically viewed as relatively benign in terms of side effects, aside from the known potential of NSAIDs to elicit gastrointestinal issues and/or affect kidney function with long-term dosing regimens. Thus, given the widespread availability and routine use of NSAIDs in patients, such a dosing regimen was considered justified. The concerning nature of the findings regarding carprofen co-administration with antibiotic therapy, itself a fundamental mainstay of ODRI treatment, suggested that further studies are warranted to elucidate the mechanisms underlying this interaction (specifically, the impact of selective COX-1 versus COX-2 inhibitors on bone responses following infection) to minimise the risk of impaired healing responses with NSAID use in ODRI patients.

\section{Conclusion}

The study characterised the impact of administration of a preferential COX-2 inhibitory NSAID, carprofen, on the course of a $S$. epidermidis implant-related infection and the subsequent response to antibiotic therapy. A marked inhibitory effect of carprofen on bone-forming reparative responses to $S$. epidermidis infection was confirmed and a marked reduction in antibiotic efficacy was revealed when co-administered with preferential COX-2 inhibitory NSAID regimens. Given the widespread use of NSAIDs in the general population, confirmatory studies are necessary to determine whether NSAID use is contraindicated in ODRI patients and the role of COX-1 vs. COX-2 selectivity in this process.

\section{Acknowledgements}

The authors would like to thank all the staff of the Preclinical Facility at AO Research Institute Davos,
Switzerland for their help in conducting this study. Iris Keller and Pamela Furlong are also thanked for technical support provided during the project. Invibio Biomaterials Ltd. is thanked for supplying the PEEK. This work was supported by AOTrauma as part of the CPP Bone Infection.

No conflicts of interest are noted in relation to the study.

\section{References}

Annamanedi M, Kalle AM (2014) Celecoxib sensitizes Staphylococcus aureus to antibiotics in macrophages by modulating SIRT1. PLoS One 9: e99285. DOI: 10.1371/journal.pone.0099285.

Blackwell KA, Raisz LG, Pilbeam CC (2010) Prostaglandins in bone: bad cop, good cop? Trends Endocrinol Metab 21: 294-301.

Brideau C, Van Staden C, Chan CC (2001) In vitro effects of cyclooxygenase inhibitors in whole blood of horses, dogs, and cats. Am J Vet Res 62: 1755-1760.

Chikazu D, Tomizuka K, Ogasawara T, Saijo H, Koizumi T, Mori Y, Yonehara Y, Susami T, Takato $\mathrm{T}$ (2007) Cyclooxygenase-2 activity is essential for the osseointegration of dental implants. Int J Oral Maxillofac Surg 36: 441-446.

Cook SD, Barrack RL, Dalton JE, Thomas KA, Brown TD (1995) Effects of indomethacin on biologic fixation of porous-coated titanium implants. J Arthroplasty 10: 351-358.

Croes M, Boot W, Kruyt MC, Weinans H, Pouran B, van der Helm YJM, Gawlitta D, Vogely HC, Alblas J, Dhert WJA, Öner FC (2017) Inflammation-induced osteogenesis in a rabbit tibia model. Tissue Eng Part C Methods 23: 673-685.

Croes M, Kruyt MC, Boot W, Pouran B, Braham MV, Pakpahan SA, Weinans H, Vogely HC, Fluit AC, Dhert WJ, Alblas J, Oner FC (2019) The role of bacterial stimuli in inflammation-driven bone formation. Eur Cell Mater 37: 402-419.

Cryer B, Feldman M (1998) Cyclooxygenase-1 and cyclooxygenase-2 selectivity of widely used nonsteroidal anti-inflammatory drugs. Am J Med 104: 413-421.

Depypere M, Morgenstern M, Kuehl R, Senneville E, Moriarty TF, Obremskey WT, Zimmerli W, Trampuz A, Lagrou K, Metsemakers WJ (2020) Pathogenesis and management of fracture-related infection. Clin Microbiol Infect 26: 572-578.

Einhorn TA, Gerstenfeld LC (2015) Fracture healing: mechanisms and interventions. Nat Rev Rheumatol 11: 45-54.

Eisen DP, Leder K, Woods RL, Lockery JE, McGuinness SL, Wolfe R, Pilcher D, Moore EM, Shastry A, Nelson MR, Reid CM, McNeil JJ, McBryde ES (2020) Effect of aspirin on deaths associated with sepsis in healthy older people (ANTISEPSIS): a randomised, double-blind, placebo-controlled primary prevention trial. Lancet Respir Med 9: 186195. 
Etikala A, Tattan M, Askar H, Wang HL (2019) Effects of NSAIDs on periodontal and dental implant therapy. Compend Contin Educ Dent 40: e1-e9.

Fillingham YA, Hannon CP, Roberts KC, Anesthesia A, Analgesia Clinical Practice Guideline W, Hamilton WG, Della Valle CJ (2020) Nonsteroidal anti-inflammatory drugs in total joint arthroplasty: The Clinical Practice Guidelines of the American Association of Hip and Knee Surgeons, American Society of Regional Anesthesia and Pain Medicine, American Academy of Orthopaedic Surgeons, Hip Society, and Knee Society. J Arthroplasty 35: 27042708.

Ford CA, Spoonmore TJ, Gupta MK, Duvall CL, Guelcher SA, Cassat JE (2021) Diflunisalloaded poly(propylene sulfide) nanoparticles decrease $S$. aureus-mediated bone destruction during osteomyelitis. J Orthop Res 39: 426-437

Freitag L, Gunther C, Eberli U, Furst A, Zeiter S, Stadelmann VA (2019) Relative effects of age on implant integration in a rat model: a longitudinal in vivo $\mathrm{MCT}$ study. J Orthop Res 37: 541-552.

George MD, Baker JF, Leonard CE, Mehta S, Miano TA, Hennessy S (2020) Risk of nonunion with nonselective NSAIDs, COX-2 inhibitors, and opioids. J Bone Joint Surg Am 102: 1230-1238.

Gerstenfeld LC, Einhorn TA (2004) COX inhibitors and their effects on bone healing. Expert Opin Drug Saf 3: 131-136.

Giannoudis PV, MacDonald DA, Matthews SJ, Smith RM, Furlong AJ, De Boer P (2000) Nonunion of the femoral diaphysis. The influence of reaming and non-steroidal anti-inflammatory drugs. J Bone Joint Surg Br 82: 655-658.

Gomes FI, Aragao MG, de Paulo Teixeira Pinto V, Gondim DV, Barroso FC, Silva AA, Bezerra MM, Chaves HV (2015) Effects of nonsteroidal antiinflammatory drugs on osseointegration: a review. J Oral Implantol 41: 219-230.

Hamilton SM, Bayer CR, Stevens DL, Bryant AE (2014) Effects of selective and nonselective nonsteroidal anti-inflammatory drugs on antibiotic efficacy of experimental group A streptococcal myonecrosis. J Infect Dis 209: 1429-1435.

Hartog E, Menashe O, Kler E, Yaron S (2010) Salicylate reduces the antimicrobial activity of ciprofloxacin against extracellular salmonella enterica serovar typhimurium, but not against salmonella in macrophages. J Antimicrob Chemother 65: 888-896.

Hendrix AS, Spoonmore TJ, Wilde AD, Putnam NE, Hammer ND, Snyder DJ, Guelcher SA, Skaar EP, Cassat JE (2016) Repurposing the nonsteroidal antiinflammatory drug diflunisal as an osteoprotective, antivirulence therapy for staphylococcus aureus osteomyelitis. Antimicrob Agents Chemother 60: 5322-5330.

Hsu JR, Mir H, Wally MK, Seymour RB, Orthopaedic Trauma Association Musculoskeletal Pain Task Force (2019) Clinical practice guidelines for pain management in acute musculoskeletal injury. J Orthop Trauma 33: e158-e182.
Hughes JM, McKinnon CJ, Taylor KM, Kardouni JR, Bulathsinhala L, Guerriere KI, Popp KL, Bouxsein ML, Proctor SP, Matheny RW Jr (2019) Nonsteroidal anti-inflammatory drug prescriptions are associated with increased stress fracture diagnosis in the US army population. J Bone Miner Res 34: 429-436.

Iqbal Z, Khan A, Naz A, Khan JA, Khan GS (2009) Pharmacokinetic interaction of ciprofloxacin with diclofenac: a single-dose, two-period crossover study in healthy adult volunteers. Clin Drug Investig 29: 275-281.

Jeffcoach DR, Sams VG, Lawson CM, Enderson BL, Smith ST, Kline H, Barlow PB, Wylie DR, Krumenacker LA, McMillen JC, Pyda J, Daley BJ, University of Tennessee Medical Center, Department of Surgery (2014) Nonsteroidal anti-inflammatory drugs' impact on nonunion and infection rates in long-bone fractures. J Trauma Acute Care Surg 76: 779-783.

Jiang Y, Wang SN, Wu HT, Qin HJ, Ren ML, Lin JC, Yu B (2019) Aspirin alleviates orthopedic implant-associated infection. Int J Mol Med 44: 12811288.

Kaneko H, Mehrotra M, Alander C, Lerner U, Pilbeam C, Raisz L (2007) Effects of prostaglandin E2 and lipopolysaccharide on osteoclastogenesis in RAW 264.7 cells. Prostaglandins Leukot Essent Fatty Acids 77: 181-186.

Kawaguchi H, Pilbeam CC, Harrison JR, Raisz LG (1995) The role of prostaglandins in the regulation of bone metabolism. Clin Orthop Relat Res: 36-46.

Khodaverdian V, Pesho M, Truitt B, Bollinger L, Patel P, Nithianantham S, Yu G, Delaney E, Jankowsky E, Shoham M (2013) Discovery of antivirulence agents against methicillin-resistant Staphylococcus aureus. Antimicrob Agents Chemother 57: 3645-3652.

Kidd LJ, Cowling NR, Wu AC, Kelly WL, Forwood MR (2013) Selective and non-selective cyclooxygenase inhibitors delay stress fracture healing in the rat ulna. J Orthop Res 31: 235-242.

Kortram K, Bezstarosti H, Metsemakers WJ, Raschke MJ, Van Lieshout EMM, Verhofstad MHJ (2017) Risk factors for infectious complications after open fractures; a systematic review and metaanalysis. Int Orthop 41: 1965-1982.

Laudy AE, Mrowka A, Krajewska J, Tyski S (2016) The influence of efflux pump inhibitors on the activity of non-antibiotic NSAIDS against gram-negative rods. PLoS One 11: e0147131. DOI: 10.1371/journal. pone. 0147131 .

Legras A, Giraudeau B, Jonville-Bera AP, Camus C, Francois B, Runge I, Kouatchet A, Veinstein A, Tayoro J, Villers D, Autret-Leca E (2009) A multicentre case-control study of nonsteroidal anti-inflammatory drugs as a risk factor for severe sepsis and septic shock. Crit Care 13: R43. DOI: 10.1186/cc7766.

Li C, Renz N, Trampuz A (2018) Management of periprosthetic joint infection. Hip Pelvis 30: 138-146.

Mandell BF (1999) COX 2-selective NSAIDs: biology, promises, and concerns. Cleve Clin J Med 66: 285-292. 
Marquez-Lara A, Hutchinson ID, Nunez F Jr, Smith TL, Miller AN (2016) Nonsteroidal antiinflammatory drugs and bone-healing: a systematic review of research quality. JBJS Rev 4. 01874474201603000-00005. DOI: 10.2106/JBJS.RVW.O.00055.

Metsemakers WJ (2020) Nonsteroidal antiinflammatory drugs and fracture nonunion: an ongoing debate: commentary on an article by Michael D. George, MD, MSCE, et al.: "Risk of nonunion with nonselective NSAIDs, COX-2 inhibitors, and opioids". J Bone Joint Surg Am 102: e82. DOI: 10.2106/ JBJS.20.00836.

Ong CK, Lirk P, Tan CH, Seymour RA (2007) An evidence-based update on nonsteroidal antiinflammatory drugs. Clin Med Res 5: 19-34.

Persson PE, Nilsson OS, Berggren AM (2005) Do non-steroidal anti-inflammatory drugs cause endoprosthetic loosening? A 10-year follow-up of a randomized trial on ibuprofen for prevention of heterotopic ossification after hip arthroplasty. Acta Orthop 76: 735-740.

Poultsides LA, Liaropoulos LL, Malizos KN (2010) The socioeconomic impact of musculoskeletal infections. J Bone Joint Surg Am 92: e13. DOI: 10.2106/ JBJS.I.01131.

Price CT, Kaatz GW, Gustafson JE (2002) The multidrug efflux pump NorA is not required for salicylate-induced reduction in drug accumulation by Staphylococcus aureus. Int J Antimicrob Agents 20: 206-213.

Puetzler J, Zalavras C, Moriarty TF, Verhofstad MHJ, Kates SL, Raschke MJ, Rosslenbroich S, Metsemakers WJ (2019) Clinical practice in prevention of fracture-related infection: an international survey among 1197 orthopaedic trauma surgeons. Injury 50: 1208-1215.

RadiZA (2009) Pathophysiology of cyclooxygenase inhibition in animal models. Toxicol Pathol 37: 34-46.

Riordan JT, Muthaiyan A, Van Voorhies W, Price CT, Graham JE, Wilkinson BJ, Gustafson JE (2007) Response of Staphylococcus aureus to salicylate challenge. J Bacteriol 189: 220-227.

Rissing JP, Buxton TB (1986) Effect of ibuprofen on gross pathology, bacterial count, and levels of prostaglandin E2 in experimental staphylococcal osteomyelitis. J Infect Dis 154: 627-630.

Shen Z, Pu XY, Zhang Q (2011) Salicylate functions as an efflux pump inducer and promotes the emergence of fluoroquinolone-resistant Campylobacter jejuni mutants. Appl Environ Microbiol 77: 7128-7133.

Stadelmann VA, Potapova I, Camenisch K, Nehrbass D, Richards RG, Moriarty TF (2015) In vivo $\mu \mathrm{CT}$ monitoring of osteomyelitis in a rat model. Biomed Res Int 2015: 587857. DOI: 10.1155/2015/587857.

Stadelmann VA, Thompson K, Zeiter S, Camenisch K, Styger U, Patrick S, McDowell A, Nehrbass D, Richards RG, Moriarty TF (2020) Longitudinal timelapse in vivo micro-CT reveals differential patterns of peri-implant bone changes after subclinical bacterial infection in a rat model. Sci Rep 10: 20901. DOI: 10.1038/s41598-020-77770-z.

Thompson K, Freitag L, Styger U, Camenisch K, Zeiter S, Arens D, Geoff Richards R, Fintan Moriarty T, Stadelmann VA (2020) Impact of low bone mass and anti-resorptive therapy on antibiotic efficacy in a rat model of orthopedic device-related infection. J Orthop Res 39: 415-425.

Trancik T, Mills W, Vinson N (1989) The effect of indomethacin, aspirin, and ibuprofen on bone ingrowth into a porous-coated implant. Clin Orthop Relat Res: 113-121.

Uckay I, Pittet D, Vaudaux P, Sax H, Lew D, Waldvogel F (2009) Foreign body infections due to Staphylococcus epidermidis. Ann Med 41: 109-119.

Varma GYN, Kummari G, Paik P, Kalle AM (2019) Celecoxib potentiates antibiotic uptake by altering membrane potential and permeability in Staphylococcus aureus. J Antimicrob Chemother 74: 3462-3472.

VersalovicJ, Koeuth T, LupskiJR(1991)Distribution of repetitive DNA sequences in eubacteria and application to fingerprinting of bacterial genomes. Nucleic Acids Res 19: 6823-6831.

Winnett B, Tenenbaum HC, Ganss B, Jokstad A (2016) Perioperative use of non-steroidal antiinflammatory drugs might impair dental implant osseointegration. Clin Oral Implants Res 27: e1-7.

Wurnig C, Schwameis E, Bitzan P, Kainberger F (1999) Six-year results of a cementless stem with prophylaxis against heterotopic bone. Clin Orthop Relat Res: 150-158.

Zimmerli W, Waldvogel FA, Vaudaux P, Nydegger UE (1982) Pathogenesis of foreign body infection: description and characteristics of an animal model. J Infect Dis 146: 487-497.

Zimmermann P, Curtis N (2018) The effect of aspirin on antibiotic susceptibility. Expert Opin Ther Targets 22: 967-972.

Zura R, Mehta S, Della Rocca GJ, Steen RG (2016) Biological risk factors for nonunion of bone fracture. JBJS Rev 4. 01874474-201601000-00005. DOI: 10.2106/ JBJS.RVW.O.00008.

\section{Web Reference}

1. www.easyipl.com [03.06.2021]

\section{Discussion with Reviewers}

William T. Obremskey: Will those finding be duplicated in another animal model?

Authors: It would be of interest to investigate the impact of selective COX-1 and COX-2 inhibitors in this model, to confirm which specific COX isoform underlies the mechanistic effects observed with $S$. epidermidis. Subsequent studies to investigate whether the same observations hold true with other common 
but under-studied ODRI bacterial species, such as Cuteibacterium acnes and Escherichia coli, could also reveal important insights into the role of chronic NSAID administration in the context of ODRI. Applying these findings in the context of a fracture model could also yield important insights into ODRI and how different mechanical fixation environments may impact on the process. Concerning the use of other animal models, we feel that, given its low cost, the rat model is useful to investigate the mechanistic aspects (role of COX-1 vs. COX-2 selectivity) and to optimise dosing and duration. However, testing in a larger-animal model (pigs or sheep) would ideally be performed, e.g. with a clinically-relevant plate fixation, to confirm the relevance of the findings from the rodent model.

Reviewer 1: What would be a follow-up study that would further improve our knowledge on this topic?
Authors: Given the clinical literature suggesting an impact of COX-2 inhibition on bone healing, it would be of interest to use the same animal model and compare bone responses after treatment with selective COX-1 vs. COX-2 inhibitors. These initial studies, performed in the absence vs. presence of bacterial infection, could confirm the role of COX selectivity, which could then be further evaluated in the context of a fracture/osteosynthesis model in the absence or presence of infection. In addition, such an approach could reveal appropriate durations of NSAID treatment capable of preserving the beneficial aspect of a NSAID treatment (e.g. reduced osteolysis) whilst limiting its detrimental effects (e.g. inhibitory effects on reparative bone formation).

Editor's note: The Guest Editor responsible for this paper was Willem-Jan Metsemakers. 